\title{
Zero-M-Cordial Labeling of Some Graphs
}

\author{
Freeda Selvanayagom, Robinson S. Chellathurai
}

Department of Mathematics, Scott Christian College, Nagercoil, India

Email: freedasam1969@gmail.com,robinchel@rediffmail.com

Received August 14, 2012; revised September 28, 2012; accepted October 5, 2012

\begin{abstract}
In this paper we prove that the complete bipartite graph $k_{m, n}$ where $\mathrm{m}$ and $\mathrm{n}$ are even, join of two cycle graphs $c_{n}$ and $c_{m}$ where $n+m \equiv 0 \quad(\bmod 4)$, split graph of $c_{n}$ for even " $n$ ", $K_{n} \times P_{2}$ where $n$ is even are admits a Zero-M-Cordial labeling. Further we prove that $K_{n} \times P_{2} B_{n}=K_{1, n} \times P_{2}$, of odd $n$ admits a Zero-M-Cordial labeling.
\end{abstract}

Keywords: Zero-M-Cordial Labeling; Split Graphs; Cartesian Product; H-Cordial

\section{Introduction}

We begin with finite, connected and undirected graph $G$ $=(V(G), E(G)$. If the vertices of the graph are assigned values subject to certain conditions then it is known as graph labeling. Any graph labeling will have the following three common characteristics. A set of numbers from which Vertex labels are chosen; $v_{f}(i)=$ number of vertices of $G$ having label $i$ under $f$

$e_{f}(i)=$ number of edges of $G$ having label $i$ under $f^{*}$.

The concept of cordial labeling was introduced by I. Cahit, who called a graph $\mathrm{G}$ is Cordial if there is a vertex labeling $f: v(G) \rightarrow\{0,1\}$ such that the induced labeling $f^{*}: E(G) \rightarrow\{0,1\}$, defined by

$f^{*}(x y)=|f(x)-f(y)|$, for all edges $x y \in E(G)$ and with the following inequalities holding and

$\left|v_{f}(0)-v_{f}(1)\right| \leq 1$ and $\left|e_{f}(0)-e_{f}(1)\right| \leq 1$.

In [1] introduced the concept of H-Cordial labeling. Cahit calls a graph H-Cordial if it is possible to label the edges with the numbers from the set $\{1,-1\}$ in such a way that, for some $k$, at each vertex $\mathrm{v}$ the sum of the labels on the edges incident with $\mathrm{v}$ is either $k$ or $-k$ and the inequalities $\left|v_{f}(k)-v_{f}(-k)\right| \leq 1$ and

$\left|e_{f}(1)-e_{f}(-1)\right| \leq 1$ are also satisfied where $v(i)$ and $e(j)$ are respectively, the number of vertices labeled with $i$ and the number of edges labeled with $j$. He calls a graph $\mathrm{H}_{\mathrm{n}}$-Cordial if it is possible to label the edges with the numbers from the set $\{ \pm 1, \pm 2, \cdots, \pm n\}$ in such a way that, at each vertex $v$ the sum of the labels on the edges incident with $\mathrm{v}$ is in the set $\{ \pm 1, \pm 2, \cdots, \pm n\}$ and the inequalities $\left|v_{f}(i)-v_{f}(-i)\right| \leq 1$ and $\left|e_{f}(i)-e_{f}(-i)\right| \leq 1$ are also satisfied for each $i$ with $1 \leq i \leq n$. The concept of
Zero-M-Cordial labeling is defined in [2]. A labeling $f$ of a graph $\mathrm{G}$ is called Zero-M-Cordial, if for each vertex $v$, $f(v)=0$. A graph $\mathrm{G}$ is called to be Zero-M-Cordial, if it admits a Zero-M-Cordial labeling. The usefulness of the above definition appears when one tries to find an $\mathrm{H}$ Cordial labeling for a given graph $\mathrm{G}$. If $\mathrm{H}$ is a ZeroM-Cordial subgraph of $G$ then $H$-Cordiality of $G \backslash E(H)$ simply implies H-Cordiality G.

In [1] proved that $k_{n, n}$ is H-Cordial if and only if $n>2$ and " $n$ " is even; and $k_{m, n}, m \neq n$ is H-Cordial if and only if $n \equiv 0(\bmod 4), m$ is even and $m>2, n>2$.

In [2] proved that $k_{n}$ is H-Cordial if and only if $n \equiv 0$ or $3(\bmod 4)$ and $n \neq 3$. $W_{n}$ is H-Cordial if and only if $\mathrm{n}$ is odd. $k_{n}$ is not $\mathrm{H} 2$-Cordial if $n \equiv 1(\bmod 4)$. Also [2] prove that every wheel has an $\mathrm{H}_{2}$-Cordial labeling.

In [3] several variations of graph labeling such as graceful, bigraceful, harmonious, cordial, equitable, humming etc. have been introduced by several authors. For definitions and terminologies in graph theory we refer to [4].

In this paper we investigate Zero-M-Cordial labeling on some Cartesian product of graphs, join of two graphs, and bipartite graph.

1.1. Definition: The join $G=G_{1}+G_{2}$ of graph $G_{1}$ and $G_{2}$ with disjoint point sets $V_{1}$ and $V_{2}$ and edge sets $E_{1}$ and $E_{2}$ denoted by $G=G_{1}+G_{2}$ is the graph union $G_{1} \cup G_{2}$ together with all the edges joining $v_{1}, v_{2}$. If $G_{1}$ is $\left(p_{1}, q_{1}\right)$ graph and $G_{2}$ is $\left(p_{2}, q_{2}\right)$ graph then $G_{1}+G_{2}$ is a $\left(p_{1}+p_{2}, q_{1}+q_{2}+p_{1} p_{2}\right)$.

1.2. Definition: Let $G_{1}=\left(V_{1}, E_{1}\right)$ and $G_{2}=\left(V_{2}, E_{2}\right)$ be two graphs. The Cartesian product of $G_{1}$ and $G_{2}$ which is denoted by $G_{1} \times G_{2}$ is the graph with vertex set $v=v_{1} \times v_{2}$ consisting of vertices $V=\left\{u=\left(u_{1}, u_{2}\right), v=\left(v_{1}, v_{2}\right) / u\right.$ and $v$ are adjacent in $G_{1} \times G_{2}$ whenever $u_{1}=v_{1}$ and $u_{2}$ adjacent to $v_{2}$ or $u_{1}$ adjacent to $v_{1}$ and $\left.u_{2}=v_{2}\right\}$. 
1.3. Definition: For a graph $G$ the split graph is obtained by adding to each vertex $v$, a new vertex $v^{\prime}$ such that $v^{\prime}$ is adjacent to every vertex that is adjacent to $v$ in $G$. The resultant graph is denoted by $\operatorname{spl}(G)$.

\section{Main Results}

Theorem 2.1: Every cycle $C_{n}$ of even order admits a Zero-M-Cordial labeling

Proof: Let $v_{1}, v_{2}, \cdots, v_{n}$ be the vertices of cycle $C_{n}$

Define $f: E(G) \rightarrow\{1,-1\}$ two cases are to be considered.

Case (i) $n \equiv 2(\bmod 4)$

For $1 \leq i \leq n$

$$
f\left(v_{i}, v_{i+1}\right)=\left\{\begin{array}{l}
=1, \text { if } i \equiv 1(\bmod 2) \\
=-1, \text { if } i \equiv 0(\bmod 2) \text { and } 1 \leq i \leq n
\end{array}\right.
$$

In view of the above labeling pattern we give the proof as follows:

When $n \equiv 2(\bmod 4)$

The total number of edges labeled with $-1^{\prime} s$ are given by $e_{f}(-1)=n / 2$ and the total number of edges labeled with $1^{\prime}$ s are given by $e_{f}(1)=n / 2$. Therefore the total difference between the edges labeled with $-1^{\prime} \mathrm{s}$ and $1^{\prime} \mathrm{s}$ are given by $\left|e_{f}(1)-e_{f}(-1)\right|=0$. The induced vertex labels are equal to zero. Thus for each vertex $v$, $f(v)=0$ and $\left|e_{f}(1)-e_{f}(-1)\right| \leq 1$.

Case (ii) $n \equiv 0(\bmod 4)$

The total number of edges labeled with $-1^{\prime} \mathrm{s}$ are given by $e_{f}(-1)=n / 2$ and the total number of edges labeled with $1^{\prime}$ s are given by $e_{f}(1)=n / 2$. Therefore the total difference between the edges labeled with $-1^{\prime} s$ and $1^{\prime} s$ are given by $\left|e_{f}(1)-e_{f}(-1)\right|=0$. The induced vertex labels are equal to zero. Thus for each vertex $\mathrm{v}$,

$f(v)=0$ and $\left|e_{f}(1)-e_{f}(-1)\right| \leq 1$.

Hence the cycle graph $c_{n}$, even $n$ admits a zero-Mcordial labeling.

The vertex and the edge conditions are given in Table 1. The illustration is given in Figures $\mathbf{1}$ and $\mathbf{2}$.

In Figure 1 illustrates the Zero-M-Cordial labeling for the cycle graph $C_{6}$. Among the six edges three edges receive the label +1 and the other three edges receive the label -1. In Figure 2 illustrates the Zero-M-Cordial labeling for the cycle graph $C_{8}$. Among the eight edges four edges receive the label +1 and the other four edges receive the label -1 .

Theorem 2.2: The complete bipartite graph $k_{m, n}$ admits a Zero-M-Cordial labeling for all $\mathrm{m}, \mathrm{n}$ such that $m+n$ $\equiv 0,2(\bmod 4)$.

Proof: Let $v_{1}, v_{2}, \cdots, v_{m}$ and $u_{1}, u_{2}, \cdots, u_{n}$ are the vertex set of the bipartite graph $k_{m, n}$. The number of vertices and the edges of $k_{m, n}$ is $m+n$ and $m n$ respectively.

Define $f: E(G) \rightarrow\{1,-1\}$
Table 1. The vertex and the edge conditions of cycle graph $C_{n}$.

\begin{tabular}{ccc}
\hline$n$ & Vertex condition & Edge condition \\
\hline$n \equiv 2(\bmod 4)$ & $f(v)=0$ & $e_{f}(1)=e_{f}(-1)=n / 2$ \\
$n \equiv 2(\bmod 4)$ & $f(v)=0$ & $e_{f}(1)=e_{f}(-1)=n / 2$ \\
\hline
\end{tabular}

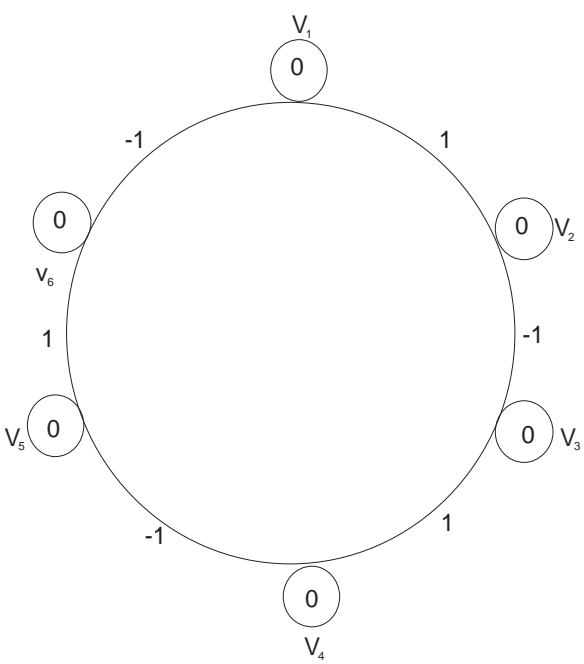

Figure 1. Zero-M-Cordial labeling on $C_{6}$.

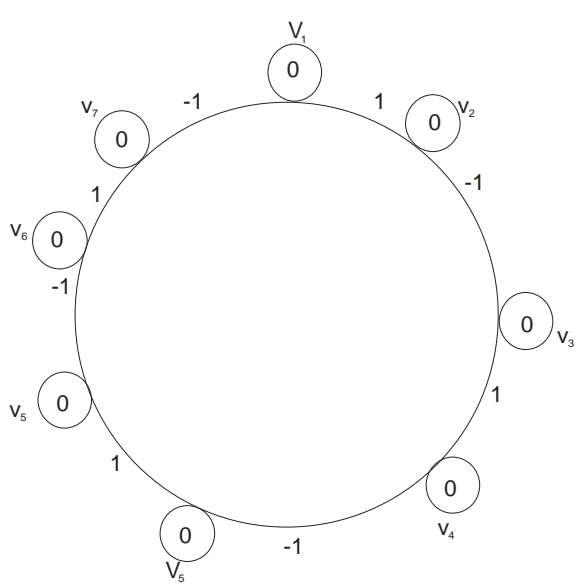

Figure 2. Zero-M-Cordial labeling on $C_{8}$.

The edge matrix of $k_{m, n}$ is given in Table 2.

In view of the above edge matrix we give the proof as follows.

Case (i) when $m+n \equiv 0(\bmod 4), m=n$.

Consider the bipartite graph $k_{4,4}$.

Using Table 2 the edge label matrix of $k_{4,4}$ is given by

\begin{tabular}{|c|c|c|c|c|}
\hline & $u_{1}$ & $u_{2}$ & $u_{3}$ & $u_{4}$ \\
\hline$v_{1}$ & -1 & 1 & -1 & \\
\hline$v_{2}$ & 1 & -1 & 1 & -1 \\
\hline$v_{3}$ & -1 & 1 & -1 & 1 \\
\hline & 1 & -1 & 1 & -1 \\
\hline & 0 & 0 & 0 & 0 \\
\hline
\end{tabular}


Table 2. Edge matrix of $\boldsymbol{k}_{m, n}$.

\begin{tabular}{|c|c|c|c|c|c|}
\hline & $u_{1}$ & $u_{2}$ & $\ldots$ & $u_{n}$ & \\
\hline$v_{1}$ & $v_{1} u_{1}$ & $v_{1} u_{2}$ & $\ldots$ & $v_{1} u_{m}$ & $v_{1}$ \\
\hline$v_{2}$ & $v_{2} u_{1}$ & $v_{2} u_{2}$ & $\ldots$ & $v_{2} u_{m}$ & $v_{2}$ \\
\hline : & : & : & & : & : \\
\hline : & : & : & & : & : \\
\hline \multirow[t]{2}{*}{$v_{m}$} & $v_{m} u_{1}$ & $v_{m} u_{2}$ & $\cdots$ & $v_{m} u_{n}$ & $v_{m}$ \\
\hline & $u_{1}$ & $u_{2}$ & $\ldots$ & $u_{n}$ & \\
\hline
\end{tabular}

With respect to the above labeling the total number of edges labeled with $1^{\prime s}$ and $-1^{\prime s}$ are given by

$e_{f}(1)=n / 2$ and $e_{f}(-1)=n / 2$. Therefore the total difference between the edges labeled with $-1^{\prime s}$ and $1^{\prime s}$ are given by $\left|e_{f}(1)-e_{f}(-1)\right|=0$. The induced vertex labels are equal to zero. Thus for each vertex $v$, $f(v)=0$ and $\left|e_{f}(1)-e_{f}(-1)\right| \leq 1$.

Hence the bipartite graph $K_{4,4}$ admits a Zero-M-Cordial labeling. The vertex and the edge conditions are given in Table 3. The illustration is given in Figure 3.

Figure 3 illustrates the Zero-M-Cordial labeling on $K_{4,4}$. Among the Sixteen edges eight edges receive the label +1 and the other eight edges receive the label -1 .

Case (ii) When $m+n \equiv 2(\bmod 4), m \neq n$.

Consider the bipartite graph $K_{2,4}$.

Using Table 2 the edge label matrix is given by

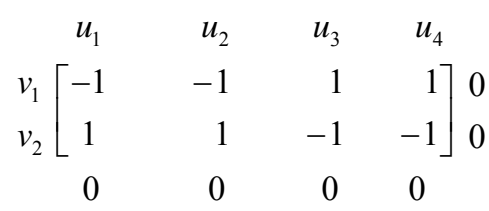

With respect to the above labeling the total number of edges labeled with $1^{\prime s}$ and $-1^{\prime s}$ are given by

$e_{f}(1)=n / 2, e_{f}(-1)=n / 2$. Therefore the total difference between the edges labeled with $-1^{\prime s}$ and $1^{\prime s}$ are given by $\left|e_{f}(1)-e_{f}(-1)\right|=0$. The induced vertex labels are equal to zero. Thus for each vertex $v, f(v)=0$ and $\left|e_{f}(1)-e_{f}(-1)\right| \leq 1$.

Hence the bipartite graph $k_{2,4}$ admits a zero-M-cordial labeling.

Figure 4 illustrates the Zero-M-Cordial labeling on $k_{2,4}$. Among eight edges four edges receive the label +1 and other four edges receive the label -1 .

Case (iii) When $m+n \equiv 0(\bmod 4)$ and $m \neq n$.

Consider the bipartite graph $k_{2,6}$.

Using Table 2 the edge label matrix is given by

$$
\begin{aligned}
& \begin{array}{llllll}
u_{1} & u_{2} & u_{3} & u_{4} & u_{5} & u_{6}
\end{array} \\
& v_{1}\left[\begin{array}{lrrrrr}
1 & -1 & 1 & -1 & 1 & -1 \\
v_{2} & 1 & -1 & 1 & -1 & 1
\end{array}\right] 0 \\
& \begin{array}{llllll}
0 & 0 & 0 & 0 & 0 & 0
\end{array}
\end{aligned}
$$

With respect to the above labeling the total number of edges labeled with $1^{\prime s}$ and $-1^{\prime s}$ are given by
Table 3. The vertex and the edge conditions of the bipartite graph $K_{m, n}$.

\begin{tabular}{ccc}
\hline$n$ & $\begin{array}{c}\text { Vertex } \\
\text { condition }\end{array}$ & Edge condition \\
\hline$m+n \equiv 0(\bmod 4), m=n$ & $f(v)=0$ & $e_{f}(1)=e_{f}(-1)=n / 2$ \\
$m+n \equiv 2(\bmod 4), m \neq n$ & $f(v)=0$ & $e_{f}(1)=e_{f}(-1)=n / 2$ \\
$\begin{array}{l}m+n \equiv 0(\bmod 4), \\
\text { and } m \neq n\end{array}$ & $f(v)=0$ & $e_{f}(1)=e_{f}(-1)=n / 2$ \\
\hline
\end{tabular}

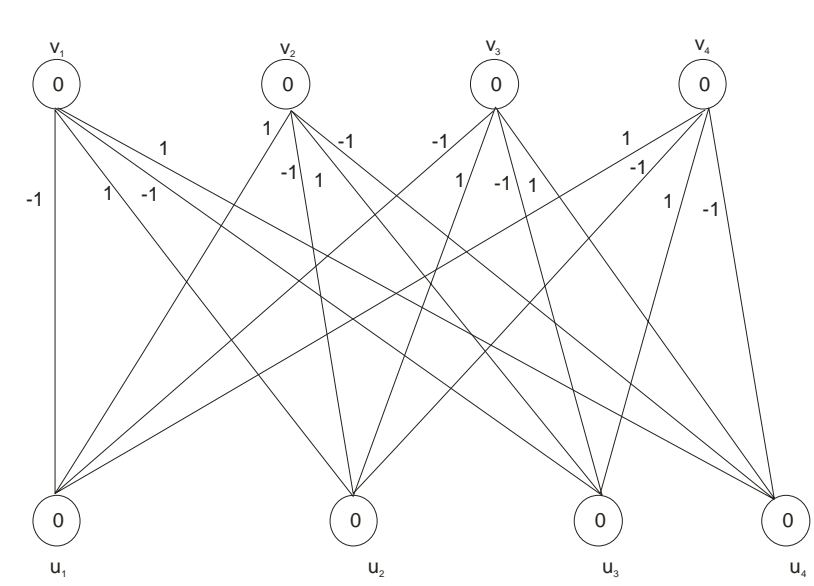

Figure 3. Zero-M-Cordial labeling on $\boldsymbol{k}_{4,4}$.

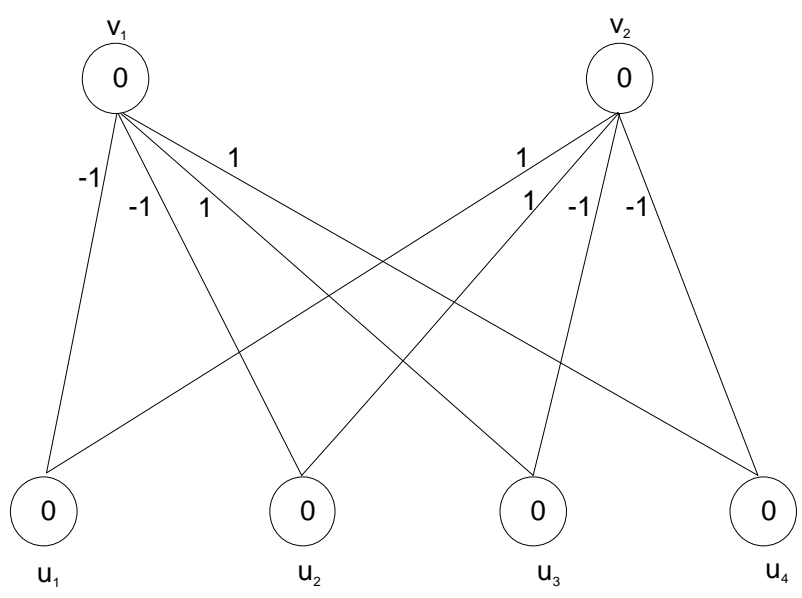

Figure 4. Zero-M-Cordial labeling on $\boldsymbol{k}_{2,4}$.

$e_{f}(1)=n / 2$ and $e_{f}(-1)=n / 2$. Therefore the total difference between the edges labeled with $-1^{\prime s}$ and $1^{\prime s}$ are given by $\left|e_{f}(1)-e_{f}(-1)\right|=0$. The induced vertex labels are equal to zero. Thus for each vertex $v$, $f(v)=0$ and $\left|e_{f}(1)-e_{f}(-1)\right| \leq 1$.

Hence the bipartite graph $k_{2,6}$ admits a Zero-M-Cordial labeling. The vertex and the edge conditions are given in Table 3.

Figure 5 illustrates the Zero-M-Cordial labeling on $k_{2,6}$. Among the Twelve edges six edges receive the label +1 and the other six edges receive the label -1 .

Theorem 2.3: The join of two cycle graphs $C_{n}$ and $C_{m}$ 


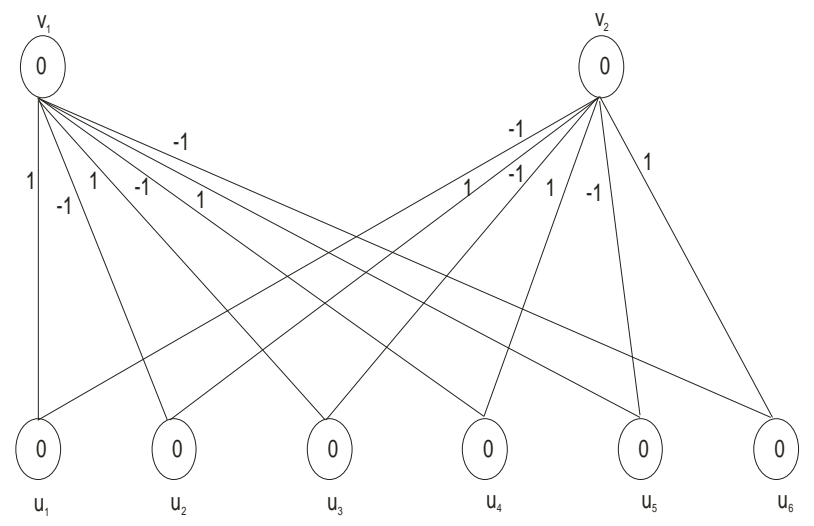

Figure 5. Zero-M-Cordial labeling on $\boldsymbol{k}_{2,6}$.

admits a Zero-M-Cordial labeling

$$
\text { if } n+m \equiv 0(\bmod 4) \text {. }
$$

Proof: Let $v_{1}, v_{2}, \cdots, v_{n}$ and $u_{1}, u_{2}, \cdots, u_{m}$ are the vertex set of the cycles $C_{n}$ and $C_{m}$. The edge set E1 and E2 is the graph union of $C_{n}$ and $C_{m}$ together with all the edges joining the vertex set $v_{1}, v_{2}, \cdots, v_{n}$ and $u_{1}, u_{2}, \cdots, u_{m}$.

We note that $|V(G)|=p_{1}+p_{2}$ and $|E(G)|=q_{1}+q_{2}+p_{1} p_{2}$

Define $f: \quad E(G) \rightarrow\{1,-1\}$

The edge matrix of $c_{n}+c_{m}$ is given in Table 4 .

In view of the above labeling pattern we give the proof as follows:

When $n+m \equiv 0(\bmod 4)$

Consider the join of two cycle graphs $C_{4}+C_{4}$. Using Table 4 the edge label matrix of $C_{4} \& C_{4}$ is given by

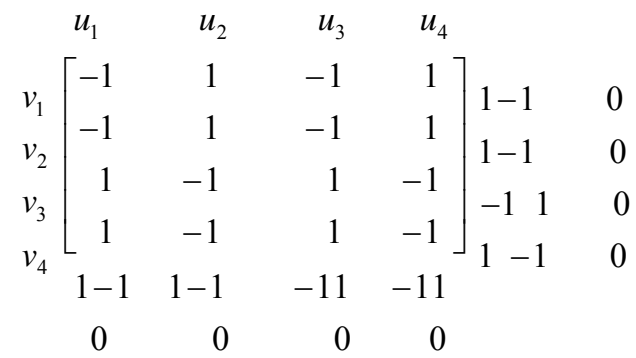

With respect to the above labeling the total number of edges labeled with $1^{\prime s}$ and $-1^{\prime s}$ are given by $e_{f}(1)=n / 2$ and $e_{f}(-1)=n / 2$. Therefore the total difference between the edges labeled with $-1^{\prime s}$ and $1^{\prime s}$ are given by $\left|e_{f}(1)-e_{f}(-1)\right|=0$. The induced vertex labels are equal to zero. Thus for each vertex $v$, $f(v)=0$ and $\left|e_{f}(1)-e_{f}(-1)\right| \leq 1$.

Hence the join of two cycle graphs $C_{4}$ and $C_{4}$ admits a Zero-M-Cordial labeling. The vertex and the edge conditions are given in Table 5.

In Figure 6 illustrates the zero-M-Cordial labeling on $c_{4}+c_{4}$. Among the twenty four edges twelve edges receive the label +1 and the other twelve edges label -1 .

Theorem 2.4: The split graph of $C_{n}$, for even $n$, admits

\begin{tabular}{|c|c|c|c|c|c|}
\hline & $u_{1}$ & $u_{2}$ & $\cdots$ & $u_{m}$ & \\
\hline$v$ & $v_{1} u_{1}$ & $v_{1} u_{2}$ & $\cdots$ & $v_{1} u_{m}$ & \\
\hline$v_{1}$ & $v_{2} u_{1}$ & $v_{2} u_{2}$ & $\cdots$ & $v_{2} u_{m}$ & $\begin{array}{ll}v_{1} v_{2}, & v_{1} v_{n} \\
v v, & v v\end{array}$ \\
\hline 2 & : & $:$ & & & $\int^{1 / 2}, 23$ \\
\hline & : & : & & & \\
\hline$V_{n}$ & $v_{n} u_{1}$ & $v_{n} u_{2}$ & $\cdots$ & $v_{n} u_{m}$ & $v_{1} v_{\mathrm{n}}, \quad v_{n-1} v_{n}$ \\
\hline & ${ }_{1} u_{2}, u_{1} u_{n}$ & $u_{1} u_{2}, u_{2}$ & $\cdots$ & $u_{m}, u_{m-1} l$ & \\
\hline
\end{tabular}

Table 4. Edge matrix of $C_{n}+C_{m}$.

Table 5. The vertex and the edge conditions of two cycle graphs $C_{n}$ and $C_{m}$.

\begin{tabular}{ccc}
\hline$C_{n}, C_{m}$ & $\begin{array}{c}\text { Vertex } \\
\text { condition }\end{array}$ & Edge condition \\
\hline$m+n \equiv 0(\bmod 4), m=n$ & $f(v)=0$ & $e_{f}(1)=e_{f}(-1)=n / 2$ \\
\hline
\end{tabular}

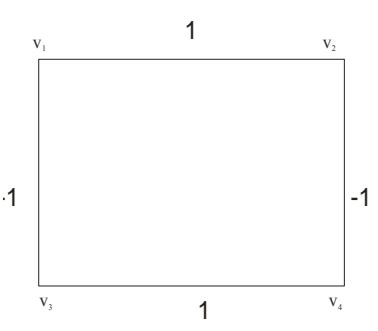

$\mathrm{C}_{4}$

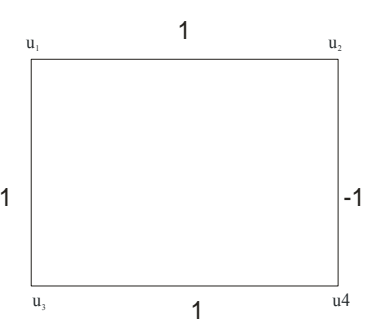

$\mathrm{C}_{4}$
$-1$

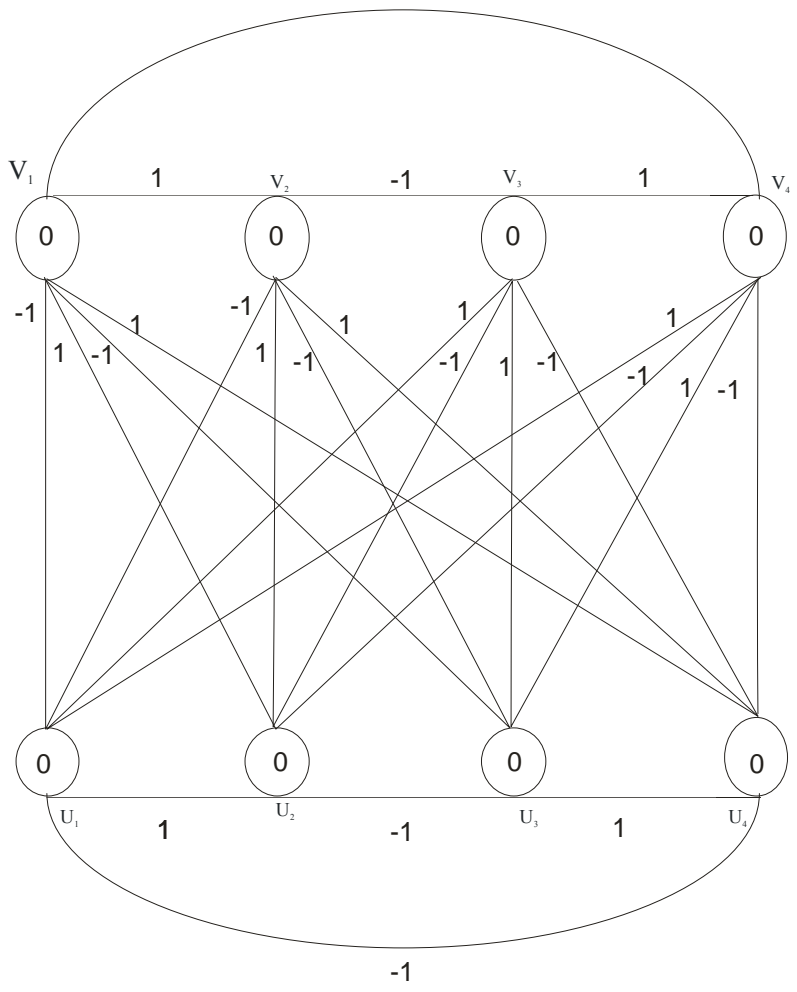

Figure 6. Zero-M-Cordial Labeling on $C_{4}+C_{4}$. 
a zero-M-cordial labeling.

\section{Proof:}

Let $v_{1}, v_{2}, \cdots, v_{n}$ be the vertices of cycle $C_{n}$ and $v_{1}^{\prime}, v_{2}^{\prime}, \cdots, v_{n}^{\prime}$ be the newly added vertices when $n$ is even.

Let $G$ be the split graph of cycle $C_{n}$ with

$$
\begin{gathered}
V(G)=\left\{v_{i}, v_{i}^{\prime}, 1 \leq i \leq n\right\}, \\
E(G)=\left\{v_{i}, v_{i+1}^{\prime}, 1 \leq i \leq n-1, v_{n} v_{1}, v_{i}^{\prime} v_{i+1},\right. \\
\\
\left.v_{n}^{\prime} v_{1}, v_{i} v_{i+1}^{\prime}, 1 \leq i \leq n-1, v_{n} v_{1}^{\prime}\right\}
\end{gathered}
$$

we note that $|V(G)|=2 n$ and $|E(G)|=3 n$.

Define $f: E(G) \rightarrow\{1,-1\}$ two cases are to be considered.

Case (i) when $n \equiv 0(\bmod 4)$

For $1 \leq i \leq n-1$

$$
\begin{aligned}
& f\left(v_{i}, v_{i+1}\right)= \begin{cases}1, & \text { if } i \text { is odd } \\
-1, & \text { if } i \text { is even }\end{cases} \\
& f\left(v_{n}, v_{1}\right)=-1
\end{aligned}
$$

For $1 \leq i \leq n-1$

$$
\begin{aligned}
& f\left(v_{i}, v_{i+1}^{\prime}\right)=-1 \\
& f\left(v_{n}, v_{1}^{\prime}\right)=-1
\end{aligned}
$$

For $1 \leq i \leq n-1$

$$
\begin{aligned}
& f\left(v_{i}^{\prime}, v_{i+1}\right)=1 \\
& f\left(v_{n}^{\prime}, v_{1}\right)=1
\end{aligned}
$$

Case (ii) when $n \equiv 2(\bmod 4)$

For $1 \leq i \leq n-1$

$$
\begin{aligned}
& f\left(v_{i}, v_{i+1}\right)=\left\{\begin{array}{l}
1, \text { if } i \equiv 1(\bmod 2) \\
-1, \text { if } i \equiv 0(\bmod 2)
\end{array}\right. \\
& f\left(v_{n}, v_{1}\right)=1
\end{aligned}
$$

For $1 \leq i \leq n-1$

$$
\begin{aligned}
& f\left(v_{i}, v_{i+1}^{\prime}\right)=-1 \\
& f\left(v_{n}, v_{1}^{\prime}\right)=-1
\end{aligned}
$$

For $1 \leq i \leq n-1$

$$
\begin{aligned}
& f\left(v_{i}^{\prime}, v_{i+1}\right)=1 \\
& f\left(v_{n}^{\prime}, v_{1}\right)=1
\end{aligned}
$$

With respect to the above labeling pattern we give the proof as follows.

The total number of edges labeled with $1^{\prime s}$ and $-1^{\prime s}$ are given by $e_{f}(1)=n$ and $e_{f}(-1)=n$. Therefore the total difference between the edges labeled with $-1^{\prime s}$ and $1^{\prime s}$ are given by $\left|e_{f}(1)-e_{f}(-1)\right|=|n-n|=0$, differ by zero. The induced vertex labels are equal to zero. Thus for each vertex $v, f(v)=0$ and $\left|e_{f}(1)-e_{f}(-1)\right| \leq 1$.
Hence the split graph of $C_{n}$ for even $\mathrm{n}$ admits a ZeroM-Cordial labeling. The vertex and the edge conditions are given in Table 6.

In Figure 7 illustrates the Zero-M-Cordial labeling on split c8. Among the twenty four edges twelve edges receive the label +1 and the other twelve edges receive the label -1 .

Theorem 2.5: $K_{n} \times P_{2}$ admits a Zero-M-Cordial labeling for even $n$.

Proof: Let $G$ be the graph $K_{n} \times P_{2}$ where $n$ is even and $V(G)=\left\{V_{i j} \quad i=1,2, \cdots, n\right.$ and $\left.j=1,2\right\} \quad$ be the vertices of the graph $G$.

We note that $|V(G)|=2 n$ and $|E(G)|=n^{2}$ as

$\left|V\left(k_{n}\right)\right|=n$ and $\left|E\left(k_{n}\right)\right|=\frac{n(n-1)}{2}$

Define $f: E(G) \rightarrow\{1,-1\}$ as follows

For $1 \leq i, k \leq n$

$$
f\left(v_{i_{1}}, v_{k_{1}}\right)=1
$$

For $n<i, k \leq n+2$

$$
f\left(v_{i_{1}}, v_{k_{1}}\right)=-1
$$

For $1 \leq i, k \leq n$

$$
f\left(v_{i_{2}}, v_{k_{2}}\right)=1
$$

For $n<i, k \leq n+2$

$$
f\left(v_{i_{2}}, v_{k_{2}}\right)=-1
$$

For $1 \leq i \leq n$

$$
f\left(v_{i_{1}}, v_{i_{2}}\right)=-1
$$

With respect to the above labeling pattern we give the proof as follows.

The total number of edges labeled with $1^{\prime s}$ and $-1^{\prime s}$ are given by $e_{f}(1)=n / 2$ and $e_{f}(-1)=n / 2$. Therefore the total difference between the edges labeled with $-1^{\prime s}$ and $1^{\prime s}$ are given by $\left|e_{f}(1)-e_{f}(-1)\right|=0$, differ by 0 . The induced vertex labels are equal to zero. Thus for each vertex $\mathrm{v}, f(v)=0$ and $\left|e_{f}(1)-e_{f}(-1)\right| \leq 1$.

Hence $K_{n} \times P_{2}$ admits a Zero-M-Cordial labeling for even $n$. The vertex and the edge conditions are given in Table 7.

Figure 8 illustrates the Zero-M-Cordial labeling on $k_{n} \times p_{2}$. Among the sixteen edges eight edges label +1 and the other eight edges label -1 .

Theorem 2.6: $W_{n} \times P_{2}$ admits a Zero-M-Cordial labeling for odd $n$.

Proof: Let $\mathrm{G}$ be the graph $W_{n} \times P_{2}$ where $\mathrm{n}$ is odd and $V(G)=\left\{V_{i j} \quad i=1,2, \cdots, n+1\right.$ and $\left.j=1,2\right\}$ be the vertices of graph $G$.

We note that $|V(G)|=2(n+1)$ and $|E(G)|=5 n+1$ as $\left|V\left(W_{n}\right)\right|=n+1$ and $\left|E\left(W_{n}\right)\right|=2 n$ 
Table 6. The vertex and the edge conditions of split of $C_{n}$.

\begin{tabular}{ccc}
\hline$C_{n}, C_{m}$ & Vertex condition & Edge condition \\
\hline$n \equiv 0(\bmod 4)$ & $f(v)=0$ & $e_{f}(1)=e_{f}(-1)=n$ \\
$n \equiv 2(\bmod 4)$ & $f(v)=0$ & $e_{f}(1)=e_{f}(-1)=n$ \\
\hline
\end{tabular}

Table 7. Vertex and the edge condition of $k_{n} \times p_{2}$.

\begin{tabular}{ccc}
\hline$n$ & Vertex condition & Edge condition \\
\hline Even & $f(v)=0$ & $e_{f}(1)=e_{f}(-1)=n / 2$ \\
\hline
\end{tabular}

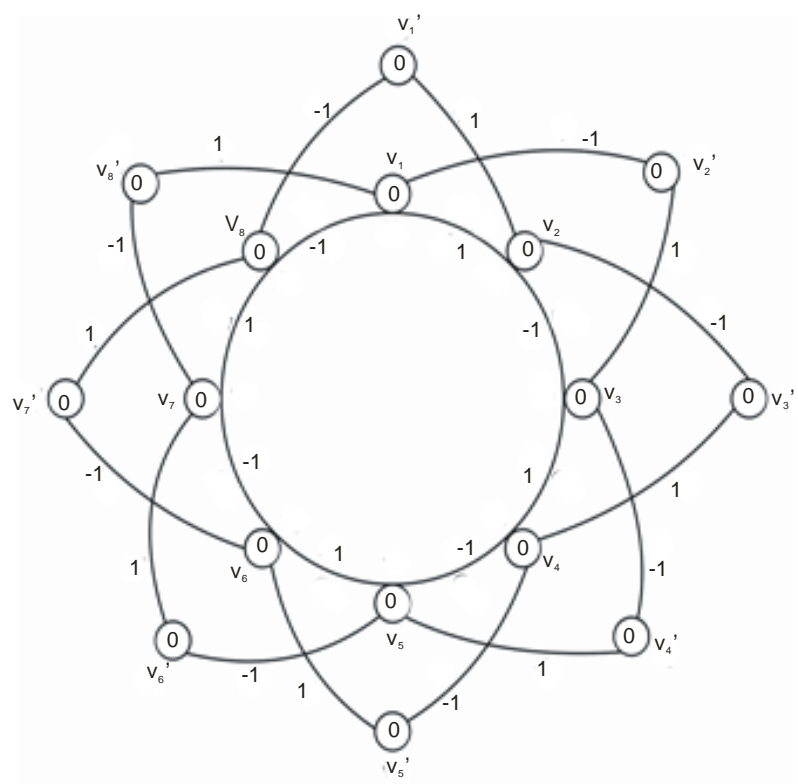

Figure 7. Zero-M-Cordial labeling on split $C_{8}$.

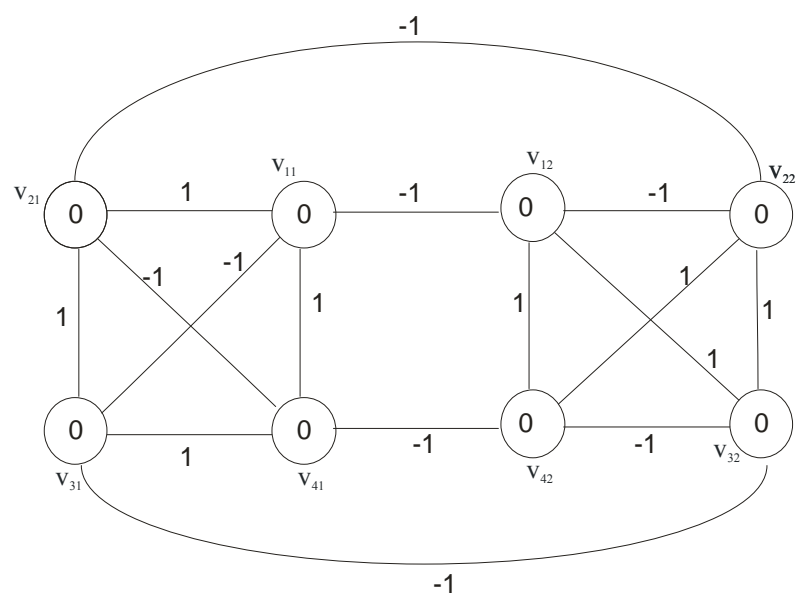

Figure 8. Zero-M-Cordial labeling on $k_{4} \times p_{2}$.

Define $f: E(G) \rightarrow\{1,-1\}$ as follows

For $1 \leq i, k \leq 2 n-2$

$f\left(v_{i_{1}}, v_{k_{1}}\right)=1$

For $2 n-2<i, k \leq 2 n$

$$
f\left(v_{i_{1}}, v_{k_{1}}\right)=-1
$$

For $1 \leq i, k \leq 2 n-2$

$f\left(v_{i_{2}}, v_{k_{2}}\right)=1$

For $2 n-2<i, k \leq 2 n$

$$
f\left(v_{i_{2}}, v_{k_{2}}\right)=-1
$$

For $1 \leq i \leq n+1$

$$
f\left(v_{i_{1}}, v_{i_{2}}\right)=-1
$$

The total number of edges labeled with $1^{\prime s}$ and $-1^{\prime s}$ are given by $e_{f}(1)=n / 2$ and $e_{f}(-1)=n / 2$. Therefore the total difference between the edges labeled with $-1^{\prime s}$ and $1^{\prime s}$ are given by $\left|e_{f}(1)-e_{f}(-1)\right|=0$, differ by 0 . The induced vertex labels are equal to zero. Thus for each vertex $v, f(v)=0$ and $\left|e_{f}(1)-e_{f}(-1)\right| \leq 1$.

Hence $W_{n} \times P_{2}$ admits a Zero-M-Cordial labeling for odd $n$. The vertex and the edge conditions are given in Table 8.

Figure 9 illustrates the Zero-M-Cordial labeling on $W_{3} \times P_{2}$. Among the sixteen edges eight edges receive the label +1 and the other eight edges label -1 .

Theorem 2.7: $B_{n}=k_{1, n} \times P_{2}$ (also known as book graph) admits a Zero-M-Cordial labeling for odd $n$.

Proof: Let $G$ be the graph $K_{1, n} \times P_{2}$ where $\mathrm{n}$ is odd and $V(G)=\left\{V_{i j} \quad i=1,2, \cdots, n+1, j=1,2\right\}$ be the vertices of $\mathrm{G}$.

We note that $|V(G)|=2(n+1)$ and $|E(G)|=3 n+1$.

Define $f: E(G) \rightarrow\{1,-1\}$ as follows

For $1 \leq i, k \leq n-1$

$$
f\left(v_{i_{1}}, v_{k_{1}}\right)=1
$$

For $n-1<i, k \leq n$

$$
f\left(v_{i_{1}}, v_{k_{1}}\right)=-1
$$

For $1 \leq i, k \leq n-1$

$$
f\left(v_{i_{2}}, v_{k_{2}}\right)=1
$$

For $n-1<i, k \leq n$

$$
f\left(v_{i_{2}}, v_{k_{2}}\right)=-1
$$

For $1 \leq i \leq n$

$$
f\left(v_{i_{1}}, v_{i_{2}}\right)=-1
$$

For $n<i \leq n+1$

$$
f\left(v_{i_{1}}, v_{i_{2}}\right)=1
$$

The total number of edges labeled with $1^{\prime s}$ and $-1^{\prime s}$ are given by $e_{f}(1)=n / 2$ and $e_{f}(-1)=n / 2$. Therefore the total difference between the edges labeled with $-1^{\prime s}$ and $1^{\prime s}$ are given by $\left|e_{f}(1)-e_{f}(-1)\right|=\left|\frac{n}{2}-\frac{n}{2}\right|=0$, differ by 0 . The induced vertex labels are equal to zero. Thus for each vertex $v, f(v)=0$ and 
Table 8. Vertex and the edge condition of $W_{n} \times P_{2}$.

\begin{tabular}{ccc}
\hline$n$ & Vertex condition & Edge condition \\
\hline Odd & $f(v)=0$ & $e_{f}(1)=e_{f}(-1)=n / 2$ \\
\hline
\end{tabular}

Table 9. Vertex and the edge condition of $B_{n}=k_{1, n} \times P_{2}$.

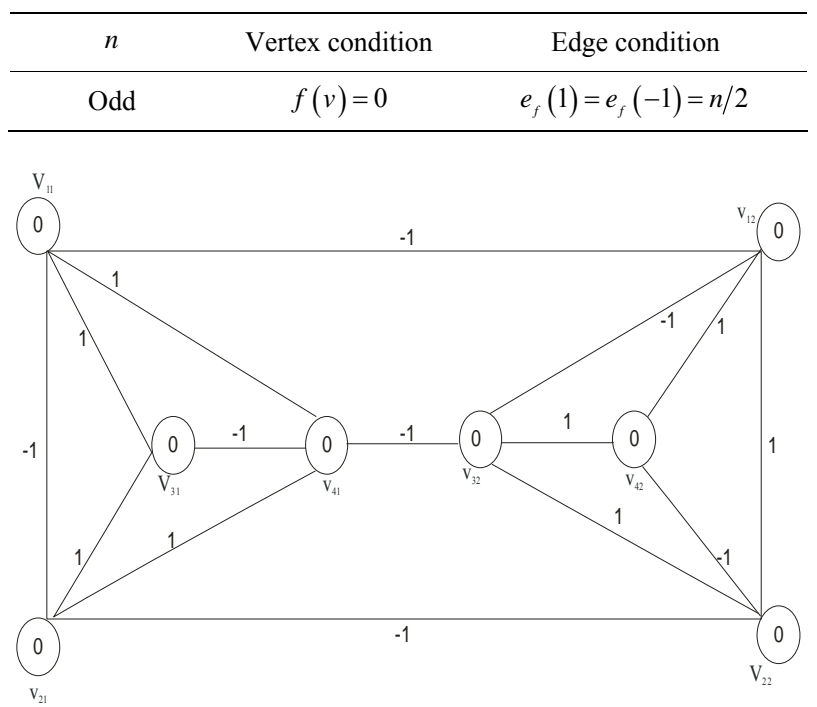

Figure 9. Zero-M-Cordial labeling on $W_{3} \times P_{2}$.

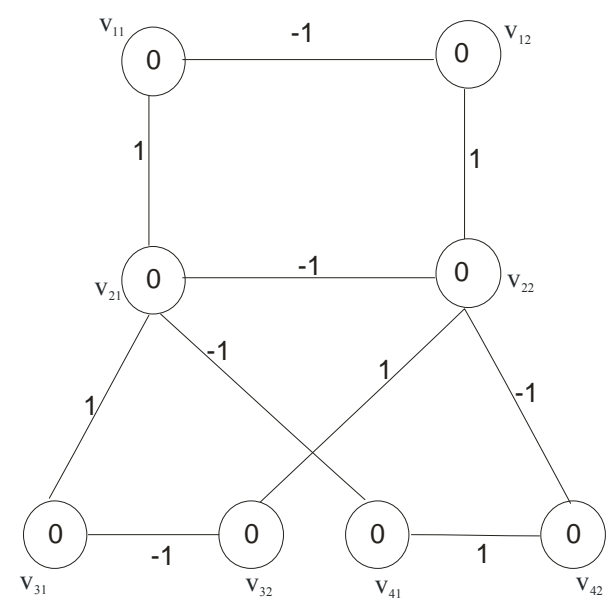

Figure 10. Zero-M-Cordial labeling on $K_{1,3} \times P_{2}$. $\left|e_{f}(1)-e_{f}(-1)\right| \leq 1$.

Hence $B_{n}=k_{1, n} \times P_{2}$ (also known as book graph) admits a Zero-M-Cordial labeling for odd $n$. The vertex and the edge conditions are given in Table 9.

Figure 10 illustrates Zero-M-Cordial labeling on $K_{1,3} \times P_{2}$. Among the ten edges five edges receive the label +1 and the other five edges receive the label -1 .

\section{Concluding Remark}

Here we investigate Zero-M-Cordial labeling for Cartesian product of some graphs, join of two cycle graphs, split graphs and bipartite graphs. Similar results can be derived for other graph families and in the context of different graph labeling problem is an open area of research.

\section{REFERENCES}

[1] I. Cahit, "H-Cordial Graphs," Bulletin of the Institute of Combinatorics and Its Applications, Vol. 18, 1996, pp. 87-101.

[2] M. Ghebleh and R. Khoeilar, "A Note on 'H-Cordial graphs,," Bulletin of the Institute of Combinatorics and Its Applications, Vol. 31, 2001, pp. 60-68.

[3] J. A. Gallian, "A Dynamic Survey of Graph Labeling," The Electronics Journal of Combinatories, Vol. 18, 2011.

[4] F. Harary, "Graph Theory," Addison Wesley, Reading, 1972. 